\title{
A CRITICAL DISCUSSION OF THE RECORDED FORM OF THE FAST VITAL CAPACITY RECORD
}

\author{
BY \\ L. BERNSTEIN \\ From the Department of Physiology, London Hospital Medical College, E.1
}

(RECEIVED FOR PUBLICATION MAY 4, 1953)

If an individual inspires as deeply as he can and then expires his whole vital capacity as rapidly as he is able into a recording spirometer the resulting record is known as a fast vital capacity (F.V.C.) tracing, or, sometimes, as an expiratory vital spirogram (E.V.S.). Recently much attention has been given to the possibility of using this record as a basis for the prediction of the maximum ventilatory capacity (M.V.C.) of the same individual, and a number of papers have appeared (Roche and Thivollet, 1949; Tiffeneau, Bousser and Drutel, 1949 ; Gaensler, 1951 ; Kennedy, 1953) in which attempts have been made to correlate the slope of the F.V.C. record-or of some portion of it-with the M.V.C. Kennedy (1953) draws attention to the form of what he terms the E.V.S. tracing and comments that it

". . . is never uniform throughout its length, and may, except in certain types of abnormal patients, be divided into two fractions. The first fraction is relatively steep and linear. It then breaks away into a second fraction, which is less steep, and later the curve becomes undulatory. The position of the dividing point between the first and second fraction, i.e., where the tracing deviates from a straight line, has been termed the critical point (Kennedy, 1950)."

D'Silva and Kazantzis, working in this department, have lately made a similar attempt to correlate the slope of the F.V.C. record with the M.V.C. In their experience (personal communication) curves of the form shown by Kennedy are never recorded. They state that the curve is one with a smoothly changing slope, always concave upwards, and that it does not have the straight portion followed by a sudden inflexion at a critical point or the subsequent region of small undulations (Kazantzis, 1953).

Clearly it is a matter of considerable importance, both for the theoretical implications and for the validation of any predictions which are to be made from such records, to decide which of these very different kinds of curve is correct, and, if possible, to explain the differences.
Kennedy (1953) states that his records were made on a spirometer almost identical with that described by Gilson and Hugh-Jones (1949). From their description, which, unfortunately, is not detailed, it appears that their spirometer was physically similar to the conventional Knipping type of spirometer. The typically smooth curves of D'Silva and Kazantzis were recorded on the improved spirometer described by Bernstein, D'Silva, and Mendel (1952). It is therefore not unreasonable to assume as a working hypothesis that the difference in the spirometer used is responsible for the difference in the records obtained.

Several records of F.V.C. expirations were made on a Knipping spirometer. These curves all resembled those described by Kennedy, and one of these records is shown at A in Fig. 1, together with, at B, a reproduction of one of Kennedy's records, for purposes of comparison. The time and volume scales for these records have been made as nearly as possible the same. It can be seen that records made on our Knipping spirometer are like those of Kennedy ; they are unlike those recorded on the Bernstein, D'Silva, and Mendel spirometer. An example of one of these is shown at $\mathrm{C}$ in Fig. 1. This supports the thesis that it is the difference in the spirometers which causes the difference in the records obtained.

It is possible to explain these differences in recordings made on the two types of spirometer in one of three ways: (1) That the Knipping spirometer records are accurate while those on the new spirometer are inaccurate; (2) that the reverse is true ; and (3) that both spirometers are inaccurate but in different ways or to differing extents. Considering the first two of these possibilities, the Knipping spirometer is known to possess considerable errors for recording tidal ventilation at respiratory rates over 35 respirations per minute (Bernstein and Mendel, 1951) whereas the new spirometer is known to be very much more 


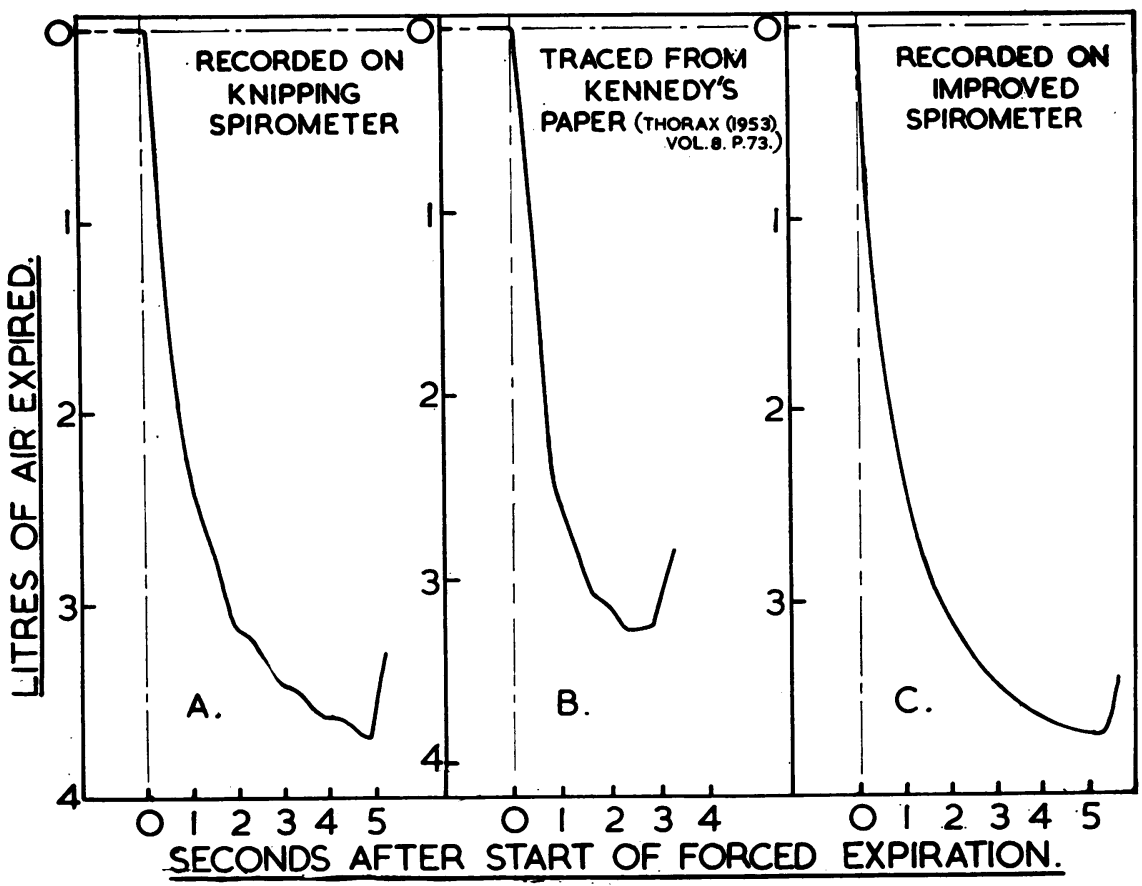

Fig. 1.-Fast vital capacity records as recorded : A, on Knipping spirometer, B, by Kennedy (1953), record traced from a published paper, $C$, on the improved spirometer when the subject was the same individual as in $\mathbf{A}$.

accurate up to much higher respiratory rates (Bernstein, D'Silva, and Mendel, 1952). It is therefore more likely in this instance that the Knipping spirometer converts an initially smooth F.V.C. curve into an undulating one than that the new spirometer converts an initially undulating F.V.C. curve into a smooth one. The latter hypothesis would presuppose that the error of the new spirometer was exactly equal and opposite to existing undulations in the F.V.C. curve-a most unlikely event. The possibility that the new spirometer merely fails to record the undulations can be rejected because it is known to have a linear response curve in the frequency range concerned. The first possibility can therefore be discarded, but it is not possible to decide between the second and third without further analysis.

Bernstein and Mendel (1951) have shown that conventional spirometers, such as the Knipping type, possess large recording errors, which are due primarily to the inertia of their moving parts. It is possible that these errors may be responsible for the differences in the records obtained when such a spirometer is used to record F.V.C. tracings. When gas is suddenly introduced into it the bell fails to move with sufficient speed and this has two consequences: (a) the spirometer under-records, and (b) the pressure rises in the be.1 and this causes a depression in the water level in the inner limb of the water jacket and an elevation in the level i⿱⺈ the outer limb.

If the introduction of the gas into the spiros. meter is stopped the water in the jacket tends tof return to its correct level, but, because of its ow inertia, it overshoots and executes an exponerg tially damped train of sinusoidal oscillations at frequency determined by the geometry of the water jacket. During these oscillations each time the level of the water rises higher in the inneP limb of the water jacket than in the outer limb air will be displaced into the bell, and this wid cause the spirometer to over-record. Conversely when the level falls lower in the inner limb thay in the outer limb the spirometer will undef record. Fig. 2 is an illustration taken from the paper of Bernstein and Mendel (1951) showing such oscillations produced by the sudden intr $\vec{\Phi}$ duction of air into, and the sudden extraction air from, a Knipping spirometer.

During the recording of a F.V.C. the rate entry of air into the spirometer initially will te 
very high, and this, as has just been described, will cause oscillations in the water jacket. The motion imposed on the spirometer bell by these oscillations will be superimposed on the motion due to the completion of the F.V.C. expiration, and this might, perhaps, explain the undulatory character of the recorded curve.

This theory of the behaviour of a Knipping spirometer affords a way of testing the second and third hypotheses (above) to explain the different F.V.C. curves obtained on the two spirometers. If the amplitude of the motion of the Knipping spirometer bell, due to oscillations of the water column, were known it would then be possible to predict the way in which a presumed accurate record on the new spirometer would be distorted by the Knipping spirometer, i.e., to predict the shape of the record on the latter instrument. Agreement between this prediction and the actual record would provide very strong confirmation of the hypothesis that the new spirometer gives an accurate record, and the Knipping spirometer an inaccurate record of the F.V.C., and would weigh against the hypothesis that both instruments were inaccurate.

Calculation of the Oscillatory Motion of the Spirometer Bell Due to Oscillations OF THE WATER COLUMN

The frequency of the water jacket oscillations during expiration, measured directly from the record reproduced in Fig. 2, was found to be about 66 cycles per minute. The amplitudes of the first and second positive half cycles were found to be $165 \mathrm{c.cm}$. and $125 \mathrm{c.cm}$. respectively. From these values it was possible to calculate that the time constant of damping of the oscillatory train (i.e., the time in which the amplitude is reduced to $\left(\mathrm{e}^{-1}\right)$ of its initial value) was approximately 1.6 seconds, and that the initial undamped amplitude was approximately $380 \mathrm{c.cm}$. peak to peak. From these figures it was then possible to calculate the amplitude and direction of the oscillation at any instant after air began to enter the spirometer. It must be emphasized that these measurements can be regarded as only approximate, because the number of oscillations was too few for great accuracy in measurement to be attained. However, the calculated values for the first three negative, and for the first three positive, peaks were compared with the measured values. The agreement was extremely good, and indicated that the calculations were not greatly in error.

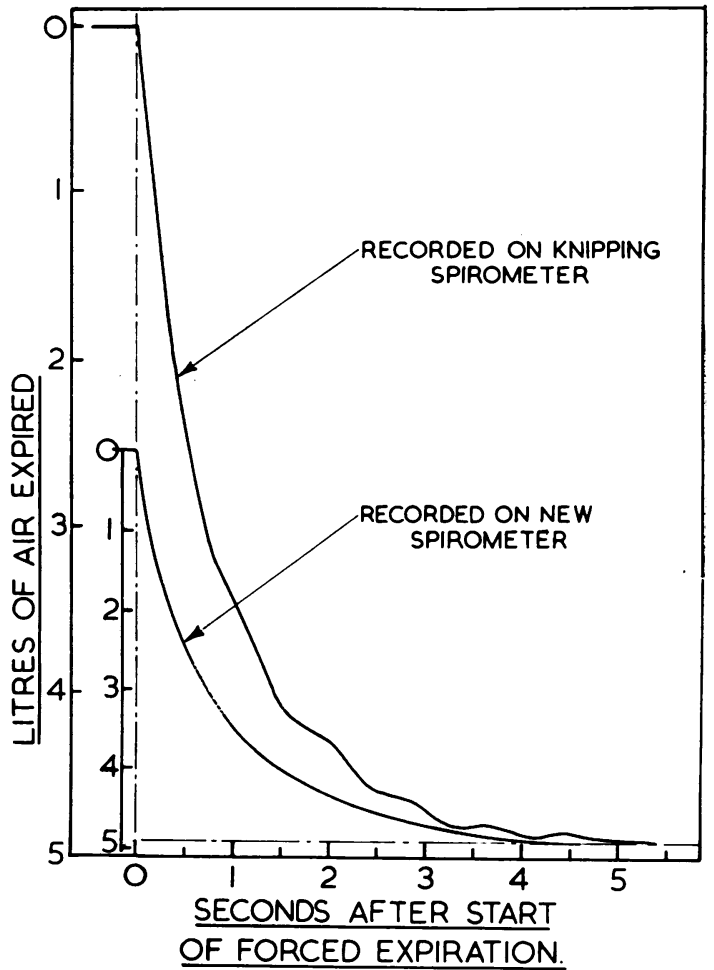

FIG. 3.-Records of fast vital capacity expirations by the same individual into a Knipping spirometer and into the improved (Bernstein, D'Silva and Mendel, 1952) spirometer. The inset (Bernstein, D'Silva and Mendel, 1952) spirometer. The inset
volume scale refers to the new spirometer: the different volume scales for the two spirometers are due to their bells having different cross-sectional areas.

\section{Prediction of a F.V.C. Record as Recorded on A KNIPPING SPIROMETER}

A human subject performed maximum effort expirations of his vital capacity into the improved spirometer described by Bernstein, D'Silva, and Mendel, and also into the Knipping spirometer. The records obtained are shown in Fig. 3. (It should be noted that the vertical scale for the improved spirometer is about half that for the Knipping spirometer.) From the curve recorded on the improved spirometer measurements were made of the volume recorded at successive 0.1 second intervals (up to 5 seconds) after the start of the F.V.C. expiration. For each time interval, this recorded value was added to the calculated value for the motion of the Knipping spirometer bell due to oscillation of the water column. The sum was regarded as a predicted value for the Knipping spirometer record at the corresponding time after the start of a F.V.C. expiration. From the series of predicted values a predicted curve 


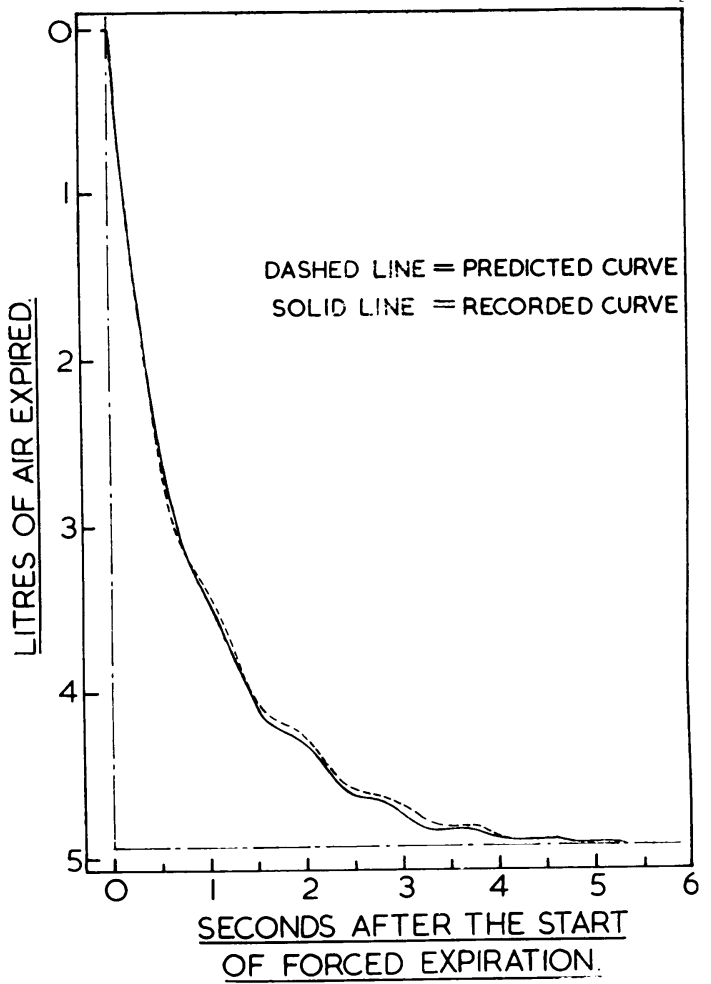

FIG. 4-Record of a fast vital capacity expiration of an individual recorded on a Knipping spirometer (solid line) and of the predicted form of this record (dashed line). The solid line curve is the same as in Fig. 3.

for the Knipping spirometer was drawn. The result is shown in Fig. 4 with the actual record made on the Knipping spirometer superimposed. The agreement is remarkably good, and such discrepancies as do exist may reasonably be attributed to the difficulties, already mentioned, in making precise measurements and calculations of the oscillatory train. The similarity to Kennedy's (1953) records is again striking.

\section{Discussion}

It therefore appears likely that the record of the F.V.C. is a smooth curve, and that the complicated records displayed by Kennedy $(1950,1953)$ are due to recording artifacts of the kind described by Bernstein and Mendel (1951). It is not immedi- $\overrightarrow{\vec{D}}$ ately apparent what implications this may have on $\bar{c}$ the conclusions drawn by Kennedy from his 음 records. It may well be that these will not be in- के validated, but both the records and the argument $\mathbb{\Phi}$ from these records will require re-examination.

Kennedy (1953) has criticized Tiffeneau, Bousser, and Drutel (1949) on the ground that their use of the volume of air expelled in the first $\overrightarrow{\vec{\omega}}$ second of a F.V.C. as a basis for predicting the $\stackrel{\omega}{\omega}$ M.V.C. is erroneous because this time extends $\vec{x}$ beyond the critical point. This criticism cannot 0 be sustained if, in fact, the critical point is only an artifact.

The results of this analysis show very forcibly응 the need to examine the accuracy of apparatus $\rightarrow$ used to record physiological phenomena before 3 accepting the recorded form of such phenomena as a true representation of fact and before reason- $\mathcal{S}$ ing too widely from the records.

\section{SUMMARY}

Evidence is presented that the form of the fast vital capacity (F.V.C.) or expiratory vital spirogram (E.V.S.) record is a smooth curve.

It is suggested that the more complicated curves $\stackrel{\odot}{\circ}$ which have been described previously are due to $\Rightarrow$ recording artifacts and that these can be eliminated 3 by the use of a well-designed spirometer.

Attention is drawn to the necessity for consider-O ing errors and artifacts due to the apparatus used in the interpretation of this kind of record.

I am indebted to Professor J. L. D'Silva and Dr. G. Kazantzis for their permission to quote from their unpublished work and to reproduce one of their records, and also to Dr. Kazantzis for his assistance in performing the experiment described in this paper?

\section{REFERENCES}

Bernstein, L., and Mendel, D. (1951). Thorax, 6, 297. D'Silva, J. L., and Mendel, D. (1952). Ibid., 7, 255. D'Silva, J. L., and Kazantzis, G. (unpublished). Personal com munication.

Gaensler, E. A. (1951). Amer. Rev. Tuberc., 64, 256.

Gilson, J. C., and Hugh-Jones, P. (1949). Clin. Sci., 7, 185.

Kazantzis, G. (1953). J. Physiol., 122, 77 .

Kennedy, M. C. S. (1950). Beitr. Silikose-Forsch., Heft 10, p. 19. Kennedy, M. C. S. (1953). Thorax, 8, 73 .

Roche, L., and Thivollet, J. (1949). Arch. Mal. prof., 10, 448.

Tiffeneau, R., Bousser, J., and Drutel, P. (1949). Paris mid. (partie mid), 39, 543.

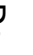

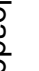

